March 10, 2018

UU-HEP/98-01

\title{
Noncommutative Gauge Theories in Matrix Theory
}

\author{
Pei-Ming $\mathrm{Ho}^{\dagger}\left[\right.$ and Yong-Shi $\mathrm{Wu}^{\ddagger}$ 巴 \\ $\dagger$ Department of Physics, University of Utah \\ Salt Lake City, Utah 84112-0830 \\ ¥ School of Natural Sciences, Institue for Advanced Study \\ Princeton, New Jersey 08540
}

\begin{abstract}
We present a general framework for Matrix theory compactified on a quotient space $\mathbf{R}^{n} / \Gamma$, with $\Gamma$ a discrete group of Euclidean motions in $\mathbf{R}^{n}$. The general solution to the quotient conditions gives a gauge theory on a noncommutative space. We characterize the resulting noncommutative gauge theory in terms of the twisted group algebra of $\Gamma$ associated with a projective regular representation. Also we show how to extend our treatments to incorporate orientifolds.
\end{abstract}

PACS numbers: 11.25.-w, 11.25.Mj, 11.25.Sq

\footnotetext{
${ }^{1}$ Present address: Department of Physics, Jadwin Hall, Princeton University, Princeton, NJ 08544.

${ }^{2}$ On sabbatical from Department of Physics, University of Utah, Salt Lake City, UT 84112-0830.
} 


\section{Introduction}

According to general relativity, classical gravity is nothing but (spacetime) geometry. It has long been suspected that quantum fluctuations of gravity near the Planck scale may make points in space fuzzy and, therefore, call for modifying our current description of geometry. Recent progress in string theory has indeed confirmed this suspicion. Facts and arguments on the lately discovered string dualities have pointed to the existence of a fundamental quantum theory in eleven dimensional spacetime, called M theory, which underlies all known five perturbative superstring theories [1]. The BFSS matrix model was proposed in ref. [2] for the microscopic description of $\mathrm{M}$ theory in discrete lightcone quantization [3], in terms of a set of $N$ partons, called D0-branes, on which strings can end. A novel feature of the $\mathrm{M}$ (atrix) theory is that the nine transverse coordinates

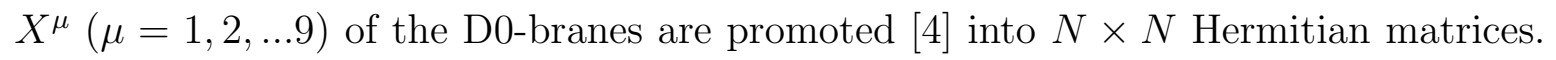
One smells the need for new geometry that deals with spaces whose coordinates (as functions on the space) are noncommutative. Such spaces are called noncommutative spaces, and their geometry noncommutative geometry (NCG).

NCG, as Connes advocated [5], deals with a geometric space not as a set of points, instead starting with the set of all functions defined on it. For usual manifolds, the latter forms a commutative algebra, from which one can reconstruct the underlying manifold, in accordance with the Gel'fand-Naímark theorem [6]. But in NCG, it can be a noncommutative algebra. The precise data for defining a noncommutative space consist of the spectral triple $(\mathcal{A}, \mathcal{H}, \mathcal{D})[5]$. Here $\mathcal{A}$ is an associative algebra, thought of as the algebra of functions (including the coordinates) on the space. $\mathcal{H}$ is a Hilbert space that represents the algebra $\mathcal{A}$ as operators acting on it, thought of as the Hilbert space on which the coordinates are represented as operators; the trace associated with the inner product of $\mathcal{H}$ can be used to define the integration on the space. Finally $\mathcal{D}$ is a derivation acting on $\mathcal{H}$, called the Dirac operator, representing the usual derivative operator.

Gauge theory on a noncommutative space will be abbreviated as noncommutative gauge theory $(\mathrm{NCGT})$. The gauge group $\mathcal{G}(\mathcal{A})$ is the group of unitary elements in $\mathcal{A}$, while the covariant derivative is obtained by adding terms of the form $\sum_{i} a_{i}\left[D, b_{i}\right]$ for $a_{i}, b_{i} \in \mathcal{A}$ to the Dirac operator. The generalized gauge potential in this form is used to incorporate the usual Higgs fields [7] in the Yang-Mills-Connes action. Previously we have shown [8] that the BFSS matrix model action, which is given by the dimensional reduction of ten dimensional supersymmetric $U(N)$ Yang-Mills theory down to $0+1$ dimension, can be understood as an NCGT: The defining algebra is $\mathcal{A}_{0}=M_{N}(\mathbf{C})$, that 
of $N \times N$ complex matrices, and the Hilbert space is $\mathcal{H}_{0}=\mathbf{C}^{N}$. The Dirac operator is simply $\mathcal{D}=\Gamma^{0}\left(\partial_{0}+A_{0}\right)+\Gamma^{\mu} X_{\mu}$. Here both the gauge potential $A_{0}$ and the "Higgs" fields $X^{\mu}(\mu=1,2 \ldots, 9)$ are the generalized gauge potentials. (In the following the explicit form of the BFSS action is not needed.)

In a recent paper [9], Matrix theory on a torus is shown to be described by NCGT on a quantum torus. A further case-by-case study is given in ref. [10]. In this note we will show that Matrix theory compactified on $\mathbf{R}^{n} / \Gamma$, with $\Gamma$ a discrete group of Euclidean motions in $\mathbf{R}^{n}$, generically leads to NCGT characterized by the group algebra of $\Gamma$ twisted by a projective regular representation. Appropriate $\mathbf{Z}_{\mathbf{2}}$-grading or $\mathbf{Z}_{\mathbf{2}}$-graded extension of $\Gamma$ will incorporate orientifolds.

\section{Quotient Conditions}

We want to study the compactification of some transverse directions on a flat quotient space $\mathbf{R}^{n} / \Gamma$, with $n \leq 9$ and $\Gamma$ a discrete group of the Euclidean motions in $\mathbf{R}^{n}$. If the action of $\Gamma$ is free (i.e. has no fixed points), then $\mathbf{R}^{n} / \Gamma$ is a manifold with $\Gamma$ as the fundamental group; otherwise it is an orbifold.

For $g \in \Gamma$, we write the action of $g$ on $\mathbf{x} \in \mathbf{R}^{n}$ as $\mathbf{x} \rightarrow \mathbf{x} g \equiv R_{g}(\mathbf{x})+T_{g}(\mathbf{x})$, where $R_{g}$ is a rotation, while $T_{g}$ a translation. For simplicity, assume $\Gamma$ preserves orientation and consider the naturally lifted action of $\Gamma$ on the matrix-valued $X^{\mu}$, denoted as $\Phi_{g}$ for $g \in \Gamma$ :

$$
\Phi_{g}^{\mu}(X)=\left(R_{g}\right)_{\nu}^{\mu} X^{\nu}+d_{g}^{\mu} \mathbf{I}
$$

$X^{\mu}$ is unchanged if it is not in the compactified directions. The superpartner $\Psi$ transforms under $\Phi_{g}$ as a ten-dimensional Majorana-Weyl spinor under the proper rotation $R_{g}$. Or one may work out the action of $\Phi_{g}$ on $\Psi$ by requiring the Matrix model action be invariant. Below we will concentrate on the bosonic variables, since the fermionic ones can be similarly treated.

To implement the compactification, we follow the techniques for dealing with Dbranes on a quotient space [11, 12, 13]. Namely for a D0-brane located at some point in $\mathbf{R}^{n} / \Gamma$, we need to consider all image D0-branes in $\mathbf{R}^{n}$ under the action of $\Gamma$, locating on a $\Gamma$-orbit. Then the (Chan-Paton) label for the D0-brane is extended from a single $i(=1,2, \ldots, N)$ to a pair $(g, i)$ with $g \in \Gamma$. The compactification (or quotient)

to $\mathbf{R}^{n} / \Gamma$ implies gauging the discrete symmetry $\Gamma$ for the D0-brane quantum mechanics, or the gauge equivalence of the open strings described by the coordinate matrix 
element $\left(X^{\mu}\right)_{\left(g_{1}, i\right),\left(g_{2}, j\right)}$ and by its image under simultaneous action of $\Gamma$ on $g_{1}$ and $g_{2}$ :

$$
\left(X^{\mu}\right)_{\left(g_{1} g, i\right),\left(g_{2} g, j\right)}=\Phi_{g}^{\mu}\left(X_{\left(g_{1}, i\right),\left(g_{2}, j\right)}\right) .
$$

We introduce a set of unitary operators $\left\{U_{g}: g \in \Gamma\right\}$ to implement the action of $\Gamma$ on the matrix variables:

$$
U_{g}^{-1} X^{\mu} U_{g}=\Phi_{g}^{\mu}(X)
$$

Then gauging the discrete symmetry $\Gamma$ can be achieved by 1 ) including $U_{g}$ 's into the theory and making them part of the gauge group, so that the physical states are invariant under $\Gamma$ and 2) extending path integral quantization to include the twisted sectors, which are represented by the solutions to the above quotient conditions (3). Note that the shift operator $U_{g}$ also admits the following interpretation in string picture: Viewed from $\mathbf{R}^{n}$, corresponding to each $U_{g}$ there is an open string stretching between a D0brane and one of its images that is labelled by $g$. Upon compactification to $\mathbf{R}^{n} / \Gamma$, it becomes a string (in the ground state) winding on the 1-cycle corresponding to $g$. As the size of $\mathbf{R}^{n} / \Gamma$ tends to zero, these winding states become massless, so we have to incorporate them into the compactified theory.

\section{Projective Representation and Twisted Group Al- gebra}

It follows from the group property of $\Phi_{g}$ in the conditions (3) that the action of $U_{g} U_{h}$ is the same as that of $U_{g h}$, so they can differ only by a phase factor:

$$
U_{g} U_{h}=q(g, h) U_{g h},
$$

with $q(g, h)=\exp \{i \alpha(g, h)\}$. Here $q(g, h)$ or $\alpha(g, h)$ depends on a pair of group elements $(g, h)$. We do not want to impose constraints more than necessary [14, the operator $U_{e}$ (corresponding to the identity $e$ of $\Gamma$ ) has to be the identity operator $\mathbf{1}$ up to a phase factor. Without loss of generality we rescale $U_{e}$ to 1 . Then it follows from eq. (田) that $q(g, e)=q(e, g)=1$. The associativity $\left(U_{f} U_{g}\right) U_{h}=U_{f}\left(U_{g} U_{h}\right)$ leads to the 2-cocycle condition:

$$
q(f, g) q(f g, h)=q(f, g h) q(g, h) .
$$

Thus, the operators $U_{g}$ 's in the quotient conditions form a faithful, projective representation of $\Gamma$, determined by a 2 -cocycle $q(g, h)$. The faithfulness implies that only 
$U_{e}$ is proportional to 1 . Physically we need this condition, in order for the quotient conditions to faithfully describe the desired compactification.

For instance, if $g$ and $h$ commute with each other: $g h=h g$, then the difference $\theta(g, h)=\alpha(g, h)-\alpha(h, g)$ is a cohomological invariant. So the projectivity condition (4) can be replaced by

$$
U_{g} U_{h}=\exp \{i \theta(g, h)\} U_{h} U_{g} .
$$

Using each $U_{g}(g \in \Gamma)$ as a basis vector, we can generate a vector space with complex coefficients, whose dimension is the order $|\Gamma|$ of the group $\Gamma$, i.e. the number of elements in $\Gamma$, which is either finite or countable. Upon introducing multiplication of two $U$ 's by eq. (1), this complex vector space is turned into an algebra, denoted as $\mathbf{C}^{\alpha} \Gamma$, called the group algebra of $\Gamma$ twisted (or deformed) by the 2-cocycle $\alpha$.

Now we come to the key point of our approach: In the spirit of NCG using an algebra to define a space, we use the twisted group algebra $\mathbf{C}^{\alpha} \Gamma$ to define a noncommutative space, and construct a Hilbert space $\mathcal{H}_{\Gamma}$ to represent the algebra. It is natural to take it to be the linear space spanned by $\left\{U_{g}\right\}$ in the projective regular representation: The $U_{g}$ 's act on $\mathbf{C}^{\alpha} \Gamma$ by multiplication. There is a one-to-one correspondence between the basis $U_{g}$ in $\mathbf{C}^{\alpha} \Gamma$ and the basis states in $\mathcal{H}_{\Gamma}$. The state corresponding to the identity operator $U_{e}$ is called the "vacuum" state, denoted as $\rangle$. Then the state corresponding to $U_{h}$ is denoted as $\left.U_{h}\right\rangle$. Now $U_{g}$ 's are represented as operators on $\mathcal{H}_{\Gamma}$ whose action is the same as their action on $\mathbf{C}^{\alpha} \Gamma$.

Moreover, we need to define an inner product in $\mathcal{H}_{\Gamma}$, which should make the operators $U_{g}$ unitary. It is easy to see that the inner product should be defined by the cyclic linear functional

$$
\left\langle U_{g}\right\rangle=\delta(g, e)
$$

where $\delta(g, e)$ is 0 if $g \neq e$, and is 1 if $g=e$. Then the trace over $\mathcal{H}_{\Gamma}$ is simply $|\Gamma|$ times this linear functional.

\section{General Solution to Quotient Conditions}

Before solving the quotient conditions, upon extending the Chan-Paton indices from $i$ to $(g, i)$, the algebra $\mathcal{A}$ of the spectral triple defining the Matrix model is enlarged to $\mathcal{A} \equiv \mathcal{O}\left(\mathcal{H}_{\Gamma}\right) \times \mathcal{A}_{0}$, where $\mathcal{O}\left(\mathcal{H}_{\Gamma}\right)$ is the algebra of operators on $\mathcal{H}_{\Gamma}$, while the gauge group is the group, $\mathcal{G}(\mathcal{A})$, of all unitary elements in the algebra $\mathcal{A}$. Our problem of Matrix theory compactification is now reduced to finding the general solution to the quotient conditions on the noncommutative space, namely to write down the general 
solution for $X^{\mu}$ 's, which are understood as operators in $\mathcal{A}$ acting on the Hilbert space $\mathcal{H} \equiv \mathcal{H}_{\Gamma} \times \mathcal{H}_{0}$.

To find the general solution, one may follow Zumino's prescription [10]. The quotient conditions imply that the action of $X$ on the basis of $\mathcal{H}_{\Gamma}$, consisting of $U_{h}$ 's acting on the "vacuum" state $\rangle$, is determined by its action on the vacuum, which can be an arbitrary state in $\mathcal{H}$ :

$$
\left.\left.X^{\mu}\right\rangle=A^{\mu}(U)\right\rangle
$$

Here $A^{\mu}(U)=\sum_{g \in \Gamma} a^{\mu}(g) U_{g}\left(\right.$ with $\left.a^{\mu}(g) \in \mathcal{A}_{0}\right)$ is a general element of the algebra $\mathcal{A}$. Then for the state $X^{\mu} U_{h}$, one may use the quotient conditions (3) to move $X^{\mu}$ to the right, then use eq. (8) to obtain

$$
\left.\left.U_{h} \Phi_{h}^{\mu}(X)\right\rangle=U_{h}\left[\left(R_{h}\right)_{\nu}^{\mu} A^{\nu}(U)+d_{h}^{\mu}\right]\right\rangle
$$

Introducing the projective operators $P_{g}$ for $g \in \Gamma$ :

$$
\left.\left.P_{g} U_{h}\right\rangle=\delta(g, h) U_{h}\right\rangle
$$

and the elements of some "dual" algebra

$$
\tilde{U}_{h} \equiv \sum_{g \in \Gamma} U_{g} U_{h} U_{g}^{-1} P_{g}
$$

then eq. (9) can be written as

$$
\left.\left[A^{\nu}(\tilde{U}) \tilde{R}_{\nu}^{\mu}+\tilde{d}^{\mu}\right] U_{h}\right\rangle
$$

where

$$
\tilde{R}_{\nu}^{\mu} \equiv \sum_{g \in \Gamma}\left(R_{g}\right)_{\nu}^{\mu} P_{g}, \quad \tilde{d}^{\mu} \equiv \sum_{g \in \Gamma} d_{g}^{\mu} P_{g}
$$

Thus the general solution of $X$ is

$$
X^{\mu}=A^{\nu}(\tilde{U}) \tilde{R}_{\nu}^{\mu}+\tilde{d}^{\mu}
$$

All physical (gauge field) degrees of freedom in $X$ reside in the function $A^{\mu}(\tilde{U})$ defined on the dual space, which can be viewed as the generalized gauge field in NCGT.

As for $A_{0}$ and $X^{\mu}$ 's not in the compactified directions, they are invariant under $U_{g}$, so the solutions are simply $A_{0}=A_{0}(\tilde{U})$ and $X^{\mu}=X^{\mu}(\tilde{U})$. (See eq. (19) below.)

The constant operators $\tilde{R}_{\nu}^{\mu}$ and $\tilde{d}^{\mu}$ commute among themselves and satisfy

$$
\begin{gathered}
\tilde{R}_{\nu}^{\mu} \tilde{U}_{g}=\tilde{U}_{g} \tilde{R}_{\sigma}^{\mu}\left(R_{g}\right)_{\nu}^{\sigma}, \\
\tilde{d}^{\mu} \tilde{U}_{g}=\tilde{U}_{g}\left(\tilde{R}_{\nu}^{\mu} d_{g}^{\nu}+\tilde{d}^{\mu}\right) .
\end{gathered}
$$


For a group of translations, $\left(R_{g}\right)_{\nu}^{\mu}=\delta_{\nu}^{\mu}$ for all $g \in \Gamma$, so $\tilde{R}_{\nu}^{\mu}=\delta_{\nu}^{\mu}$ and eq. (16) suggests

that $\tilde{d}^{\mu}$ are derivatives with respect to the exponents of $\tilde{U}$ [9, 10. In general, the operator $\tilde{R}$ also has the interpretation of a derivative on a noncommutative space. A simple example was utilized in [7] to formulate the Higgs field in the standard model as the covariant derivative on the space of two points.

\section{The Resulting Noncommutative Gauge Theory}

To characterize the resulting theory as NCGT, let us first note that after imposing the quotient conditions, the surviving group $\mathcal{G}^{\prime}$ of (local) gauge symmetry becomes the commutant of $\mathcal{A}_{\Gamma} \equiv \mathrm{C}^{\alpha} \Gamma$ in $\mathcal{G}(\mathcal{A})$, i.e.,

$$
\mathcal{G}^{\prime}=\left\{g \in \mathcal{G}(\mathcal{A}):\left[g, U_{h}\right]=0, \forall h \in \Gamma\right\}
$$

Hence one may take the algebra in the spectral triple defining the compactified Matrix model to be the commutant of $\mathcal{A}_{\Gamma}$ in $\mathcal{A}$ :

$$
\mathcal{A}^{\prime}=\left\{a \in \mathcal{A}:\left[a, U_{h}\right]=0, \forall h \in \Gamma\right\}
$$

so that $\mathcal{G}^{\prime}$ is the group of unitary elements in $\mathcal{A}^{\prime}$.

From the general solution (14), it is easy to see that $\mathcal{A}^{\prime}=\mathcal{A}_{\Gamma}^{\prime} \times \mathcal{A}_{0}$, where $\mathcal{A}_{\Gamma}^{\prime}$ is spanned by the operators $\tilde{U}_{g}$ 's. It is easy to verify that

$$
\begin{aligned}
& {\left[\tilde{U}_{h}, U_{g}\right]=0, \quad \forall h, g \in \Gamma,} \\
& \tilde{U}_{g} \tilde{U}_{h}=e^{i \alpha(h, g)} \tilde{U}_{h g} .
\end{aligned}
$$

Thus $\mathcal{A}_{\Gamma}^{\prime}$ is isomorphic to the algebra obtained from $\mathcal{A}_{\Gamma}$ by reversing the ordering of all products. There is also a one-to-one correspondence between $\mathcal{H}_{\Gamma}$ and $\mathcal{A}_{\Gamma}^{\prime}$ given by $\left.\left.\tilde{U}_{g}\right\rangle=U_{g}\right\rangle \leftrightarrow \tilde{U}_{g}$

In the spirit of NCG, we use the algebra $\mathcal{A}_{\Gamma}^{\prime}$ and the associated Hilbert space $\mathcal{H}_{\Gamma}$ to define the noncommutative dual space for the compactified Matrix model. Both of them are characterized by a projective (including genuine) regular representation of $\Gamma$, known to be faithful.

Consider the group of elements $g \in \mathcal{G}(\mathcal{A})$ preserving the quotient conditions, i.e.

$$
g U_{h} g^{-1}=e^{i \beta(h)} U_{h},
$$

for some $\beta(h)$ for all $h \in \Gamma$. The gauge group $\mathcal{G}^{\prime}$ is the subgroup of those elements with $\beta(h)=0$ for all $h \in \Gamma$. It is easy to see that $\beta(h)$ has to be a 1 -cocycle for (21) 
to be consistent with $\mathcal{A}_{\Gamma}$. There is a one-to-one correspondence between $H^{1}(\Gamma, U(1))$ and scalings of $U_{h}$ by phase factors which can be realized as conjugation by elements in $\mathcal{G}(\mathcal{A})$. Such transformations shift the exponents of $\tilde{U}_{h}$ (and $U_{h}$ ) by constants, so $H^{1}(\Gamma, U(1))$ can be viewed as the global symmetry group of translations on the dual space. Generally any algebra automorphism of $\mathcal{A}_{\Gamma}^{\prime}$ is a global symmetry not existing before compactification.

Substituting the solution (14) into the BFSS action, we will get the (bosonic part of) action for the resulting NCGT, including deformed Yang-Mills theories and gauged sigma models (see below for examples).

\section{Examples}

\subsection{Matrix Theory on Quantum Tori}

To show how our abstract approach works in practice, let us first examine the case when $\Gamma$ is generated by $n$ translations $t_{a}$ along $d_{a}^{\mu}(\mu, a=1, \ldots, n \geq 2)$. Since $\Gamma$ is abelian, eq. (6) applies. Taking $g=t_{a}, h=t_{b}$, all nontrivial 2-cocycles of $\Gamma$ are determined by $\theta_{a b}=-\theta_{b a}=\theta\left(t_{a}, t_{b}\right) \in[0,2 \pi) . \mathcal{A}_{\Gamma}$ is generated by $U_{a} \equiv U_{t_{a}}$ satisfying $U_{a} U_{b}=\exp \left\{i \theta_{a b}\right\} U_{b} U_{a}$. The dual $\mathcal{A}_{\Gamma}^{\prime}$ is generated by $\tilde{U}_{a}$ 's, which satisfy eqs. (19) and (20): $\tilde{U}_{a} U_{b}=U_{b} \tilde{U}_{a}$ and $\tilde{U}_{a} \tilde{U}_{b}=\exp \left\{-i \theta_{a b}\right\} \tilde{U}_{b} \tilde{U}_{a}$.

These are just the quantum tori introduced in ref. [9] in a different way. So we are able to reproduce all results there. In particular, since $\tilde{R}_{\nu}^{\mu}=\delta_{\nu}^{\mu}$, eq. (16) implies that if we realize $\tilde{U}_{a}$ as the basic functions $\exp \left\{i \sigma_{a}\right\}$ on the torus with coordinates $0 \leq \sigma_{a}<2 \pi$, then $\tilde{d}^{\mu}$ are the derivatives $-i d_{a}^{\mu} \partial / \partial \sigma_{a}$. The noncommutative nature of the torus is exhibited in the unusual multiplication law for two functions, pertinent to eq. (20):

$$
\left(f_{1} \star f_{2}\right)(\sigma)=\left.\exp \left\{\frac{i}{2} \theta_{a b} \frac{\partial}{\partial \sigma_{a}} \frac{\partial}{\partial \sigma_{b}^{\prime}}\right\} f_{1}(\sigma) f_{2}\left(\sigma^{\prime}\right)\right|_{\sigma=\sigma^{\prime}} .
$$

With $\left[X^{\mu}, X^{\nu}\right]$ understood as $X^{\mu} \star X^{\nu}-X^{\nu} \star X^{\mu}$, one gets a deformed Yang-Mills theory parametrized by $\theta_{a b}$ on the torus with coordinates $\sigma_{a}[9]$.

\subsection{Matrix Theory on ALE Orbifolds}

As the second example, let us consider Matrix theory on ALE orbifolds [15, 16]. One will see how the results in type IIB theory [12, 17] are recovered. 
The ALE orbifolds are $\mathbf{C}^{2} / \Gamma$, where $\mathbf{C}^{2}$ is the complexification of $\mathbf{R}^{4}$ by defining $Z^{1}=X^{6}+i X^{7}$ and $Z^{2}=X^{8}+i X^{9}$, and $\Gamma$ is a discrete subgroup of $S U(2)$ properly acting on $\mathbf{C}^{2}$. Such subgroups have been classified by Klein in last century [18]. They are all finite. The action of $\Gamma$ on $\mathbf{C}^{2}$ is homogeneous: $\Phi_{g}(X)=R_{g}(X)$, where $R_{g}$ is the two dimensional representation of $\Gamma$ embedded in the fundamental representation of $S U(2)$.

The solutions of the matrix variables are immediately $Z^{i}=A^{j}(\tilde{U}) \tilde{R}_{j}^{i}, A_{0}=A_{0}(\tilde{U})$ and $X^{\mu}=X^{\mu}(\tilde{U})$ for $i, j=1,2$ and $\mu=1, \cdots, 5$.

For the case of $\mathcal{A}_{\Gamma}$ being the untwisted group algebra $\mathrm{C} \Gamma, \mathcal{H}_{\Gamma}$ is the genuine regular representation of $\Gamma$. The natural action of $U_{g}$ 's on $\mathcal{A}_{\Gamma}$ are represented by $|\Gamma| \times|\Gamma|$ matrices, which can be made block-diagonal so that each irreducible representation $R_{i}$ of $\Gamma$ appears as an $n_{i} \times n_{i}$ block $n_{i}$ times. In the basis where $U_{g}$ are block-diagonal, so are $A_{0}$ and $X^{\mu}$. The gauge group $\mathcal{G}^{\prime}$ is thus a product of unitary groups for each block: $F=\prod_{r} U\left(n_{r} N\right)$. It can be shown that the operators $\tilde{R}$ are determined by the well-known representation decomposition:

$$
\Phi \otimes R_{r}=\oplus_{s} a_{r s} R_{s}
$$

where $a_{r s}$ are the elements of the adjacency matrix $A$ of the simply laced extended Dynkin diagrams. Namely, $\tilde{R}$ 's connect the neighboring vertices $\left(R_{r}\right.$ and $\left.R_{s}\right)$ in the extended Dynkin diagram. So in the basis in which the regular representation is blockdiagonal, $\tilde{R}$ 's consist of off-diagonal blocks, which connect neighboring unitary groups making up the total gauge group $F$, with a structure isomorphic to the adjacency matrix $A$ for the extended Dynkin diagram. (These considerations for $\tilde{R}$ can be generalized to

projective representations for arbitrary $\Gamma$, as $R_{g}$ is always a representation of $\Gamma$ even if $d_{g}^{\mu} \neq 0$.)

After taking into account of the fermionic partners, we get hypermultiplets which transform in the fundamental representations of the unitary groups, according to the representations $\oplus a_{r s}\left(\mathbf{n}_{r}, \overline{\mathbf{n}}_{s}\right)$. Pictorially, they correspond to the links in the extended Dynkin diagram. Put everything together, the field content one obtains is the $\mathcal{N}=$ $1, D=6$ supersymmetric Yang-Mills theory dimensionally reduced to $0+1$ (or $1+1$ ) dimensions, if we start with the BFSS Matrix D0-brane (or string) theory.

\section{Orientifolds}

We may also consider actions of $\Gamma$ lifted to matrix variables other than the natural one. For instance, to extend our treatments to incorporate orientifolds, we need to consider a 
$\mathbf{Z}_{2}$-grading or a $\mathbf{Z}_{2}$-graded extension of the group $\Gamma[11]$. This means that we associate a number $n(g)=0,1$ to each element $g \in \Gamma$ so that this assignment is compatible with the product in the (extended) group:

$$
n(g)+n(h) \equiv n(g h) \quad(\bmod 2) .
$$

The quotient condition (3) for $U_{g}$ with $n(g)=0$ remains unchanged, while for $n(g)=1$ it should be modified to

$$
U_{g}^{-1} X^{\mu} U_{g}=\Phi_{g}^{\mu}\left(X^{T}\right)
$$

where $T$ denotes transposition of the matrices. This is what we want for orientifolding, because taking the transpose of the matrix variables corresponds to reversing the orientitation of the open strings connecting the D0-branes.

To put the quotient conditions for both $n(g)=0$ and $n(g)=1$ into the same form, instead of $U_{g}$ we may consider $\mathbf{U}_{g} \equiv U_{g} C^{n(g)}$, where $C$ is the complex conjugation operator. Then it is not difficult to repeat the orbifold construction above for the orientifolds by including this $\mathbf{Z}_{2}$-grading.

In terms of $\mathbf{U}_{g}$, the quotient condition is

$$
\mathbf{U}_{g}^{-1} X^{\mu} \mathbf{U}_{g}=\Phi_{g}^{\mu}(X)
$$

where the algebra of $\mathbf{U}_{g}$ is given by

$$
\mathbf{U}_{g} \mathbf{U}_{h}=e^{i \alpha(g, h)} \mathbf{U}_{g h}
$$

for some $\alpha(g, h)$. If $n(g)=1$, then $\mathbf{U}_{g} c=c^{*} \mathbf{U}_{g}$ for a complex number $c$. The associativity of the algebra of $\mathbf{U}_{g}$ implies that

$$
\begin{aligned}
\delta \alpha(f, g, h) & \equiv(-1)^{n(f)} \alpha(g, h)-\alpha(f g, h)+\alpha(f, g h)-\alpha(f, g) \\
& \equiv 0 \quad(\bmod 2 \pi)
\end{aligned}
$$

and shifting $\mathbf{U}_{g}$ by a phase factor $e^{i \beta(g)}$ implies that

$$
\alpha(g, h) \rightarrow \alpha(g, h)-\delta \beta(g, h),
$$

where $\delta \beta(g, h) \equiv(-1)^{n(g)} \beta(h)-\beta(g h)+\beta(g)$. The coboundary operator $\delta$ defines a cohomology $H^{2}(\Gamma, U(1))$, which can be viewed as the set of inequivalent consistent choices of the algebra of $\mathbf{U}_{g}$ [19]. The equivalent classes of $\alpha$ in $H^{2}(\Gamma, U(1))$ correspond to possible backgrounds for the compactification. Apparently the formulation of orbifolds can be viewed as a special case of the orientifolds with the trivial $\mathbf{Z}_{2}$-grading: $n(g)=0$ for all $g \in \Gamma$. 
Similarly we define the operators $\tilde{\mathbf{U}}_{g}$ acting on the Hilbert space spanned by $\left.\mathbf{U}_{g}\right\rangle$ :

$$
\left.\left.\tilde{\mathbf{U}}_{g} \mathbf{U}_{h}\right\rangle=\mathbf{U}_{h} \mathbf{U}_{g}\right\rangle
$$

and it follows that

$$
\begin{gathered}
{\left[\mathbf{U}_{g}, \tilde{\mathbf{U}}_{h}\right]=0,} \\
\tilde{\mathbf{U}}_{g} \tilde{\mathbf{U}}_{h}=\tilde{\mathbf{U}}_{h g} e^{i \alpha(h, g) \epsilon},
\end{gathered}
$$

where $\epsilon=(-1)^{\hat{n}}$ and $\left.\left.\hat{n} \tilde{\mathbf{U}}_{g}\right\rangle=n(g) \tilde{\mathbf{U}}_{g}\right\rangle$. For $\left(R_{g}\right)_{\nu}^{\mu}, d_{g}^{\mu}$ being real, the solution of $X^{\mu}$ to the quotient condition is

$$
X^{\mu}=\left(A^{\nu}(\tilde{\mathbf{U}})(1-\hat{n})+A^{\nu *}(\tilde{\mathbf{U}}) \hat{n}\right) \tilde{R}_{\nu}^{\mu}+\tilde{d}^{\mu}
$$

where $\tilde{R}_{\nu}^{\mu}$ and $\tilde{d}^{\mu}$ are still defined by (13), but now the projection operator $P_{g}$ is defined by $\left.\left.P_{g} \mathbf{U}_{h}\right\rangle=\delta(g, h) \mathbf{U}_{h}\right\rangle$. Several examples of this general solution were presented in ref. [10]. Here the new insight provided by the present treatment is that M(atrix) theory compactified on orientifolds also corresponds to noncommutative gauge theory.

\section{Presentation of the group $\Gamma$}

In the above, we have worked with all elements of $\Gamma$; however, in practice it may be more convenient to work with a presentation (caution: not representation!) of the discrete group $\Gamma$. By presentation we mean a finite set of generators $g_{a}$ 's $(a=1,2, \ldots, r)$ and a finite set of defining relations, $R: f_{m}\left(g_{1}, \ldots, g_{r}\right)=e,(1 \leq m \leq k)$, such that $\Gamma$ is isomorphic to the group $F$ freely generated by $g_{a}$ quotient by the equivalence relations $R$. Then an arbitrary element $g$ of $\Gamma$ can be written as a product of the generators $g_{a}$, with the relations $R$ understood.

If a presentation of $\Gamma$ is known, we only need to write down the quotient conditions for the generators, with corresponding operators $U_{a} \equiv U_{g_{a}}$. Also for a 2-cocycle, we only need to introduce phase factors for pairs of generators:

$$
U_{a} U_{b}=q_{a, b} U_{a b}, \quad\left|q_{a, b}\right|=1,
$$

or equivalently a phase factor for each defining relation: each $f_{m}\left(g_{1}, \ldots, g_{r}\right)=e$ gives rise to

$$
f_{m}\left(U_{1}, \ldots, U_{r}\right)=p_{m} \mathbf{1}, \quad\left|p_{m}\right|=1
$$

For instance, if $U_{a}$ commutes with $U_{b}$, one may replace eq. (34) with

$$
U_{a} U_{b}=\exp \left\{i \theta_{a b}\right\} U_{b} U_{a}
$$


where $\theta_{a b}$ is antisymmetric. Working only with generators or with defining relations simplifies the job of finding all possible 2-cocycles.

We can generate the twisted group algebra, $\mathcal{A}_{\Gamma}$, and the representation Hilbert space, $\mathcal{H}_{\Gamma}$, in terms of the generators $U_{a}$ 's. Following the above procedure, one can solve the $X_{i}$ 's in the quotient conditions in terms of $\tilde{U}_{a}$ 's (or $\tilde{\mathbf{U}}_{a}$ 's), which can be viewed as a set of coordinates on the dual space. Examples presented in ref. [10] were worked out explicitly in details in this way.

So the use of presentation is technically very helpful. However, the presentation of a given group $\Gamma$ may not be unique. We would like to emphasize that the underlying mathematics and physics are independent of the choice of a presentation. In particular it is possible that different choices of generators in $\Gamma$ can lead to essentially the same set of "deformed" defining relations (35), when there is a corresponding algebra automorphism on $\mathcal{A}_{\Gamma}^{\prime}$ (or $\mathcal{A}_{\Gamma}$ ). This can be understood as a global symmetry on the dual space where the generators of $\mathcal{A}_{\Gamma}^{\prime}$ can be interpreted as coordinates.

\section{Discussions}

To conclude, the following remarks are in order.

1) When the fermionic field $\Psi$ is taken into account, the group $\Gamma$ generically will be extended into a larger group acting on a superspace. Since the spinor representation of spatial rotations is a double covering of the vector representation, the 2-cocycle $\alpha(g, h)$ may include the operator $i \pi F$, where $F$ is the fermion number operator. The Dirac operator acting on $\Psi$ is then given by $\Gamma^{0} D_{0}+\Gamma^{\mu} X_{\mu}$ with $A_{0}$ and $X_{\mu}$ the general solution to the quotient conditions.

2) In Matrix theory, there should be many NCGT's resulting from compactification on flat quotients $\mathbf{R}^{n} / \Gamma$, with $\Gamma$ being a point group or space group in $\mathbf{R}^{n}$ (for $2 \leq n \leq 9$ ) and allowing a nontrivial 2-cocycle.

3) Our approach can be easily used to construct the quotient Matrix theory on $\mathcal{M} / \Gamma$, if Matrix theory on $\mathcal{M}$ is known and has a discrete symmetry $\Gamma$ of $\mathcal{M}$. It also

applies to compactification of any other matrix models, such as the IKKT matrix model for IIB strings [20]. 


\section{Acknowledgement}

P.M.H. thanks Yi-Yen Wu for discussion. Work was supported in part by NSF grant No. PHY-9601277 and a grant from Monell Foundation.

\section{References}

[1] For a review, see, e.g., J.H. Schwarz, Phys. Lett. B367, 97 (1996); M.J. Duff, Int. J. Mod. Phys. A11, 5623 (1996); P.K. Townsend, hep-th/9612121; and the paper of E. Witten, Nucl. Phys. B443, 85 (1995).

[2] T. Banks, W. Fischler, S.H. Shenker, L. Susskind, Phys. Rev. D55, 112 (1997).

[3] L. Susskind, hep-th/9704080.

[4] E. Witten, Nucl. Phys. B460, 335 (1996).

[5] A. Connes, "Noncommutative Geometry", Academic Press, 1994.

[6] I.M. Gel'fand, M.A. Naímark, Mat. Sbor. 12, 197 (1943).

[7] A. Connes, J. Lott, Nucl. Phys. Proc. Suppl. 18B, 29 (1991).

[8] P.-M. Ho, Y.-S. Wu, Phys. Lett. B398: 251 (1997).

[9] A. Connes, M.R. Douglas, A. Schwarz, hep-th/9711162.

[10] P.-M. Ho, Y.-Y. Wu, Y.-S. Wu, hep-th/9712201.

[11] E.G. Gimon, J. Polchinski, Phys. Rev. D54, 1667 (1996).

[12] M.R. Douglas, G. Moore, hep-th/9603167.

[13] W. Taylor, IV, Phys. Lett. B394, 283 (1997).

[14] In string theory there should be no pure gauge twist [11].

[15] M.R. Douglas, JHEP 07 (1997) 004; M.R. Douglas, H. Ooguri, S.H. Shenker: Phys. Lett. B402, 36 (1997).

[16] D. Berenstein, R. Corrado, J. Distler, hep-th/9712049. 
[17] C.V. Johnson, R.C. Myers, Phys. Rev. D55, 6382 (1997).

[18] F. Klein, "Lectures on the Icosahedron and the Solution of an Equation of Fifth Degree", Dover, New York, 1913.

[19] P.-M. Ho, "Twisted Bundles on Quantum Torus and BPS States in Matrix Theory", hep-th/9803166.

[20] N. Ishibashi, H. Kawai, Y. Kitazawa, A. Tsuchiya, Nucl. Phys. 498, 467 (1997). 